\title{
Kollektive Intelligenz und Psychologische Sicherheit: Haben wir Intelligenz im Gefühl?
}

\author{
Birgit Feldhusen
}

Eingegangen: 18. April 2021 / Angenommen: 26. April 2021 / Online publiziert: 3. August 2021

(C) Der/die Autor(en) 2021

Zusammenfassung Psychologische Sicherheit und Kollektive Intelligenz sind zwei Konzepte, die sich bislang in der Erforschung von Gruppenlernen und Kollaboration als förderlich erwiesen haben. Ersteres steht für ein gutes Teamklima, in dem sich jeder einzubringen vermag, Letzteres steht für die Fähigkeit einer Gruppe, in immer wieder neuen Umfeldern Lösungen und Lernen hervorzubringen. Überraschenderweise besitzen beide keinen förderlichen Einfluss aufeinander. Sie wirken auf die Arbeits- und Lernfähigkeit von Gruppen über zwei unterschiedliche Pfade, die einander kompensieren, aber nicht stärken können. Beide verlangen einen Reifeprozess in der menschlichen Interaktion, jedoch aus zwei unterschiedlichen Perspektiven: einer ich-zentrierten und einer situations-zentrierten bzw. selbst-transzendierenden Perspektive.

Schlüsselwörter Kollektive Intelligenz · Psychologische Sicherheit · Agile Organisation

Dr. Birgit Feldhusen $(\bowtie)$

Danube University Krems University for Continuing Education,

Dr.-Karl-Dorrek-Strasse 30, 3500 Krems, Österreich

E-Mail: Birgit.feldhusen@donau-uni.ac.at 


\title{
Collective intelligence and psychological safety: Can groups feel intelligently?
}

\begin{abstract}
Psychological Safety and Collective Intelligence have both proven to foster group learning, collaboration and team performance. Psychological Safety denotes a team climate where everybody feels safe to participate; collective intelligence describes a group's ability to collaborate and apply knowledge to adapt to a wide variety of changing tasks. Surprisingly, they do not have any provable impact on each other. Their impact on performance improvement is via two different pathways that may compensate but cannot influence each other. Both require an increased quality in human interaction, however from two different perspectives: from an ego-centered on one hand and from a situation-centered, or self-transcending, perspective on the other hand.
\end{abstract}

Keywords Collective Intelligence $\cdot$ Psychological Safety $\cdot$ Agile Organizations

Der drastische Anstieg an Volatilität und Komplexität im Organisationsumfeld erfordert immer flexiblere und reaktionsfähigere, also ,agile“ Formen der Organisation und der Zusammenarbeit. Diese sind nicht nur mit technologischem, sondern auch mit menschlichem Wandel verbunden, denn sie entfalten ihre Wirksamkeit nicht allein über die Anwendung bestimmter Tools und Methoden, sondern vor allem über die Veränderung oft jahrelang erprobter Verhaltensweisen und Begegnungsformen. Eine Strategie für erfolgreiches organisationales Handeln in einer zunehmend komplexen und dynamischen Umgebung ist die Nutzung Kollektiver Intelligenz. Dieser Artikel systematisiert die Konzepte Kollektiver Intelligenz, diskutiert den Zusammenhang ihrer Einflussfaktoren mit Psychologischer Sicherheit und leitet hieraus zwei Richtungen für menschliche Entwicklungs- und Reifeprozesse ab, die beide berücksichtigt werden müssen auf dem Weg zu smarten und agilen Organisationen.

\section{Warum wir jetzt zusammen intelligent werden sollten}

Exponentielle Entwicklungen, immer neue Aspekte und sich plötzlich ändernde Rahmenbedingungen bestimmen die Tagesordnung. Zunehmende Vernetzung und komplexe Zusammenhänge sind oft nicht vollständig zu durchschauen. Sie erfordern individuell und in der Zusammenarbeit ein „Fahren auf Sicht“, das den meisten herkömmlichen Steuerungspraktiken ihre Grenzen aufzeigt. Statt linear-kausale Zusammenhänge aufzuspüren oder zu etablieren und ihnen mit Rezepten und Best Practices zu begegnen, gilt es, Muster zu beobachten und sich Schritt für Schritt vorzutasten auf dem Weg zu neuen Lösungen. 


\subsection{Lernende Organisation, Dynamic Capabilities, Agilität: Und ewig grüßt das Murmeltier}

Eine Leitidee hat im Zusammenhang mit der sogenannten VUCA-Welt steil Karriere gemacht: Agilität. In einem umfassenden, von den Ursprüngen in der IT-Umgebung gelösten Sinne wird Agilität verstanden als ,die Fähigkeit von Teams und Organisationen, in einem unsicheren, sich verändernden und dynamischen Umfeld flexibel, anpassungsfähig und schnell zu agieren. Dazu greift Agilität auf verschiedene Methoden zurück, die es Menschen einfacher machen, sich so zu verhalten" (Hofert 2018, S. 2). Die Idee der Agilität wird oft als etwas Neues präsentiert, stützt sich jedoch auf bereits seit Jahrzehnten bekannte Ansätze. Sie erscheint als ein an die aktuellen Entwicklungen angepasstes Kondensat und Upgrade von Ideen und Gedanken der letzten Jahrzehnte, die es bisher angesichts der Beharrlichkeit von Gedankengebäuden noch nicht zur vollständigen Entfaltung und Wirksamkeit gebracht haben. Angesichts aktueller Dringlichkeiten haben sie es nun in neuem Gewand an die Spitze der Managementmoden geschafft.

Bereits Anfang der 1990er-Jahre identifizierte Peter Senge in seinem Werk „,The Fifth Discipline“ (Senge 1990) Komplexität als Treiber einer neuen Sicht auf Organisationen. Unternehmen als komplexe Systeme in einer hoch komplexen Welt könnten ihre Herausforderungen nur als permanent lernende Organisation bewältigen, die sich durch Systemdenken, d.h. zirkuläres Denken in Zusammenhängen auszeichnen. Neben dieser „Fünften Disziplin“ ging es zudem um die Meisterung weiterer vier Kerndisziplinen: Persönlichkeitsentwicklung, Mentale Modelle, gemeinsame Vision, Team-Lernen.

Ebenfalls bereits in den 1990ern etablierte Teece die bis heute vorherrschende Unternehmenstheorie der Dynamic Capabilities. Sie sieht die Fähigkeit zur kontinuierlichen, rechtzeitigen und flexiblen Anpassung der Organisation als Bedingung für nachhaltige Wettbewerbsfähigkeit (Teece und Pisano 1994, S. 1). Dynamic Capabilities beschreiben die „Adaption, Integration und Umgestaltung von internen und externen organisationalen Fertigkeiten, Ressourcen und funktionalen Kompetenzen" (Teece et al. 1997, S. 515). Nur so könnten die sich laufend verändernden Umweltund Marktbedingungen und eine unsichere Zukunft bewältigt werden. Nach Teece befinden sich Firmen und Märkte in einem laufenden Prozess der Co-Evolution. Organisationen passen sich dem Markt an und gestalten ihn gleichzeitig mit (Teece 2009, S. 11).

20 Jahre später, 2009, folgten 35 Wissenschaftler und Praktiker aus den renommiertesten Institutionen der Managementwissenschaften der Einladung Gary Hamels in das Management Lab nach Kalifornien. Sie wollten der Frage nachgehen, was es tatsächlich braucht, um Organisationen fit für die Zukunft zu machen. Für sie war Management eine ausgereifte Technologie, die sich nun für ein neues Zeitalter neu erfinden musste. Als Ergebnis formulierten sie „25 Moonshots for Management" (Hamel 2009), d.h. Landungen in Territorien außerhalb unseres bestehenden Vorstellungs- und Handlungsrahmens, die es braucht, um in der volatilen Welt von morgen bestehen zu können. Neben dem System-Denken gehören u. a. das Einlassen auf emergente Strategien, die Anbindung an einen höheren Sinn und Zweck, natürliche Hierarchien, das Verständnis von Führung als Architektur sozialer Syste- 
me, die Wertschätzung von Diversität, die Nutzung menschlicher Vorstellungskraft sowie eine Kultur des Vertrauens zu den formulierten Moonshots.

Diese wesentlichen Ideen und Konzepte finden wir in der Leitidee der Agilität gebündelt: iterative und zyklische Vorgehensweisen, konsequente Produkt-, Kunden- und Umweltorientierung, Arbeiten in kleinen, oft cross-funktionalen Teams, Transparenz und intensiver Dialog, postheroische Führung, Eigenverantwortung, konstruktive Fehlerkultur und Vertrauen. Doch seit einiger Zeit erleidet auch die Agilität das Schicksal vieler Management-Trends und wird zum Negativ-Marker. Erste Erfahrungen mit agilen Projekten konnten die hohen Erwartungen oft nicht erfüllen. Die Quoten des Scheiterns von Strategie- und/oder Change-Projekten liegen unverändert hoch, je nach Umfrage bei 50-75\% (z. B. Cândido und Santos 2015; oder Lambers 2019).

Wenn wir auf die o.g. Definition von Agilität schauen, erahnen wir den Grund: Methoden haben das Ziel, ein bestimmtes Verhalten zu erleichtern bzw. zu induzieren, können es aber nicht garantieren. Oft wird der Fokus allein auf die Methoden an sich gelegt statt auf geändertes Verhalten und Mindset in Richtung Flexibilität und Anpassungsfähigkeit. Letzteres bedarf einiges an Reife und Persönlichkeitsentwicklung und muss von jedem einzelnen Mitglied getragen werden. Mentale Modelle, Wertschätzung und Vertrauen sind an sich komplexe soziale Phänomene, die nicht durch linear-kausale, funktionale Ansätze gesteuert werden können.

\subsection{Gemeinsam schlau: Kollektiver Ausbruch aus dem Hamsterrad}

Hinweise auf die tieferliegenden, oft aus dem Blick geratenen Faktoren von Antwortfähigkeit und Agilität liefert die Forschung zu menschlicher Kollektiver Intelligenz. Die Definitionen des Begriffs der Kollektiven Intelligenz werden der Art von Verhalten gerecht, die in dynamischen, komplexen unsicheren Umfeldern gefragt ist:

Das Forschungsteam vom Center for Collective Intelligence am Massachusetts Institute of Technology (MIT) definiert zunächst Intelligenz als die Fähigkeit, zu lernen und zu verstehen, sich durch die Anwendung des eigenen Wissens an seine Umwelt anzupassen und damit mit neuen und schwierigen Situationen umzugehen (MIT 2021). Sie basiert auf der generellen kognitiven Fähigkeit, über eine Vielzahl unterschiedlichster Aufgaben hinweg immer wieder gute Ergebnisse zu erzielen (Woolley et al. 2010, S. 686). Kollektive Intelligenz wurde in der Folge definiert als das Verhalten von Personengruppen, die gemeinsam scheinbar intelligente Dinge tun, d.h. neue oder schwierige Situationen angehen, Wissen anwenden, um sich an eine veränderte Umwelt anzupassen. Im Folgenden wird die Definition von Woolley et al. (2010) für Kollektive Intelligenz übernommen als ,die generelle Fähigkeit einer Gruppe, eine große Bandbreite an Aufgaben meistern zu können“ (ebd.).

Ideen von solch selbstorganisierter Zusammenarbeit sind Teil des Mythos Agilität. Sie werden der Tatsache gerecht, dass das organisationale Wissen im Interaktionsnetzwerk der Organisation residiert. In komplexen Umfeldern können Führungskräfte nicht mehr davon ausgehen, dass sie über alle relevanten Informationen verfügen bzw. dass bei ihnen alle relevanten Informationen zusammenlaufen. Handlungsalternativen im Sinne eines situativen und inkrementellen Vorgehens können 
am besten aus dem Netzwerk heraus generiert werden kann. Als Organisation agil zu sein, heißt folglich, kollektiv intelligent zu sein, d.h. gemeinsam Möglichkeiten zu finden, die immer neuen Herausforderungen der Umwelt zu meistern.

Ulrich Weinberg, Direktor der School of Design Thinking am Hasso-Plattner-Institut in Potsdam und Mitgründer der WeQ-Foundation, prägte folgenden Vergleich: Hierarchische und funktionale Organisationsstrukturen werden dasselbe Schicksal erleiden wie der gedruckte Brockhaus. Nach 200 Jahren wurde der Druck der berühmten deutschen Enzyklopädie 2014 eingestellt, weil Wissen zunehmend und zuverlässiger in Netzwerken wie z. B. Wikipedia generiert und aktualisiert werden kann. Nach Weinberg steht Ähnliches den nach Abteilung A-Z aufgereihten Strukturen einer Organisation bevor. Sie werden einem Netzwerkdenken weichen müssen, um den WeQ, die kollektive oder organisationale Intelligenz der Organisation, freizusetzen und wettbewerbsfähig bleiben zu können (Weinberg 2018, S. 15 ff., 103 f., $177 \mathrm{f}$.).

Die Frage lautet also: Wann werden wir Menschen zusammen dümmer, wann schlauer und was heißt das für Organisationen? Kollektive Intelligenz kann beobachtet werden in Zellen, Tiergesellschaften, menschlichen Organisationen und Roboterschwärmen. In dem Versuch, das Wirkprinzip für den Menschen zu nutzen, wurden unterschiedliche Begriffe etabliert. Es entstand ein Dickicht an Schlagworten, wie Scharmintelligenz, Crowd Intelligence, Wisdom of the Crowd, die sehr unterschiedlich verstanden und genutzt werden. Im Folgenden sollen entsprechende wissenschaftliche Konzepte erläutert, abgegrenzt und hinsichtlich ihrer Aussagekraft für menschliche organisationale Intelligenz untersucht werden.

\section{Was bedeutet Kollektive Intelligenz für den Menschen?}

Obwohl die Ursprünge der Idee kollektiver kognitiver Systeme gut 120 Jahre zurückreichen, war und ist die Wissenschaft von menschlichem Geist, Kognition und Intelligenz meist noch eine Wissenschaft des Individuums. Die Idee, dass menschliche Gruppen einen Geist teilen, der Wissen und eine Form von Intelligenz trägt, ist relativ jung und speist sich aus zwei unterschiedlichen Denkrichtungen:

In der Sozialwissenschaft wurde die Diskussion um die Kollektive Psychologie Ende des 19. Jahrhunderts eröffnet: Gustave LeBon sprach von der Dummheit der Massen, die sich in primitivem und destruktivem Verhalten ausdrückt, während Emile Durkheim den Einklang funktionierender Gruppen als Basis einer Vielzahl an kulturellen Errungenschaften sah. Lévys „Collective Intelligence“ (1994) und Surowieckis „Wisdom of Crowds“ (2004) gehen auf diesen sozialwissenschaftlichen Ursprung zurück. Die Idee der Schwarmintelligenz geht hingegen zurück auf den Biologen Wheeler, der 1911 erstmals Insektengesellschaften als „Superorganismen“ bezeichnete und ihnen psychologische Eigenschaften zusprach. Die Übertragung dieses Begriffs in die Organisationswissenschaft ist vornehmlich Bonabeau zu verdanken, der untersuchte, welche Aussagen sich für menschliches organisationales Verhalten ableiten können (Bonabeau und Meyer 2001). Im Folgenden werden diese drei Konzepte näher betrachtet und ihre Aussagen für menschliche Kollektive Intelligenz und Agilität untersucht. 


\subsection{Wisdom of Crowds}

James Surowiecki (2004) untersuchte die Aggregation von Information und Kompetenzen in Kollektiven, indem er Einzelpersonen zu beliebigen Schätzfragen befragen ließ und das Ergebnis als Mittelwert aller Einzelschätzungen ermittelte. Es zeigte sich, dass die Ergebnisse sehr nah an die tatsächlichen Zahlen heranreichten. Surowiecki kam zu dem Schluss, dass in bestimmten Kontexten für die optimale Urteilsund Entscheidungsfindung keine Experten nötig sind. Eine Gruppe von durchschnittlichen Menschen kann unter bestimmten Umständen bessere Ergebnisse erzielen als einzelne Individuen innerhalb der Gruppe - selbst wenn eines der Individuen intelligenter ist als jedes andere Gruppenmitglied. Beste kollektive Entscheidungen seien demzufolge nicht durch Konsens oder Kompromiss, sondern durch Vielfalt der im Wettbewerb voneinander unabhängigen Sichtweisen erzielbar. Wesentliche Enabler, also Ermöglichungsfaktoren hierfür, seien Meinungsvielfalt, Unabhängigkeit und Dezentralität innerhalb einer Gruppe (Leimeister 2010, S. 239).

Lorenz et al. (2011) untermauerten die Ergebnisse zu Unabhängigkeit und Dezentralität, indem sie die Wirkung des „Sozialen Einflusses“ bei solchen Befragungen aufzeigten: Wussten die Teilnehmer/innen um die Antworten der anderen Teilnehmer/innen, zeigten sich diverse Verzerrungsmuster, und die individuellen Schätzungen näherten sich über mehrere Schätzrunden untereinander an, ohne dass sie treffgenauer wurden. Das Wissen um die Schätzwerte der anderen verengt offenbar die Diversität der Meinungen in solchem Ausmaß, dass der kollektive Fehler nicht ausgeglichen, geschweige denn verbessert wird (,,social influence effect"). Das Wissen um die Konvergenz der Schätzungen verstärkt schließlich die Zuversicht der einzelnen Personen in ihre (tatsächlich unzutreffender werdenden) Schätzungen (,,confidence effect“).

Hieraus folgt jedoch keinesfalls, dass soziale Interaktion kontraproduktiv für menschliche Kollektive Intelligenz sei. Schauen wir auf das Studiendesign, sehen wir, dass die Personen isoliert voneinander über Bildschirme befragt und über monetäre Anreize zur Teilnahme motiviert wurden. Sie saßen getrennt voneinander in Arbeitskabinen ohne jeglichen Kontakt, weder visuell noch verbal oder virtuell. Die Schätzungen waren über Computer abzugeben. Es gab keine soziale Interaktion oder Vermittlung eines geteilten Zieles. Der Begriff „Sozialer Einfluss“ bezog sich hier lediglich auf Bereitstellung von Informationen über Antworten anderer Personen, die auf dem eigenen Bildschirm erschienen. Unter diesen Bedingungen können wir die „Weisheit der Vielen“ daher als eine mathematische Funktion bzw. als statistisches Phänomen entlarven, wonach menschliche Schätzungen als eine Art Zufallsgenerator fungieren. Der Durchschnitt isolierter Schätz- oder Zufallsresultate kommt der Wahrheit umso näher, je größer die Reichweite der Befragung. Reichweite kann somit Experten ersetzen.

Diese Art der Wissens- oder Entscheidungsgenerierung wird heute in Form von Crowd Intelligence genutzt. Das in der „Crowd“, also einer breiten Masse vorhandene, aber dezentrale Wissen wird über Internet-Plattformen abgerufen, verbunden und zentralisiert, sodass es für bestimmte Fragestellungen genutzt werden kann. Unternehmen nutzen dies zur Entscheidungsunterstïtzung (Einbindung von Außenstehenden in Ideengenerierung und Beurteilung), in Form von Open Innovation (aktive 
strategische Nutzung der Außenwelt zur Vergrößerung des unternehmenseigenen Innovationspotenzials, z. B. Lego) oder als Crowd Sourcing (Auslagerung von Unternehmensaufgaben auf eine unabhängige Masse von Menschen) für Aufgaben- und Forschungsfragenbearbeitung oder Produktbewertungen (Leimeister 2010, S. 240). Dabei werden Arbeitsaufträge in kleine Einzelaufgaben heruntergebrochen und offen angeboten. Die Bearbeitung durch die diversen Angebotsnehmer erfolgt wie in den Forschungsexperimenten völlig unabhängig voneinander.

Mit menschlicher Intelligenz im Sinne der oben eingeführten Definition hat dies jedoch nur bedingt zu tun. Es geht eher um die effizientere Gestaltung und Abwicklung bekannter Prozesse als um die gemeinsame Handlung zur Bewältigung neuer, herausfordernder Situationen.

\subsection{Schwarmintelligenz}

Der Komplexitätsforscher Eric Bonabeau übertrug 2001 den biologischen Begriff der Schwarmintelligenz auf den menschlichen, organisationalen Bereich. Die wichtigste Erkenntnis aus der Schwarmintelligenz war für ihn, dass aus der Interaktion von Individuen, die einfachen Regeln folgen, komplexes kollektives Verhalten emergieren kann (Bonabeau und Meyer 2001, S. 110). Für die Vorhersage des Verhaltens solcher Gruppen böten sich daher Simulationsmodelle gut an. Für Bonabeau resultiert der Erfolg von Schwärmen auf Flexibilität, Robustheit und Selbstorganisation. Diese basieren - übertragen auf die menschliche Organisation - auf folgenden einfachen Regeln: Sie haben ein übergreifendes Ziel im Auge, jedes Mitglied setzt organisationale Ressourcen verantwortlich, d.h. wie seine eigenen ein, die Mitglieder können gegenseitig ihre unterschiedlichen Perspektiven wahrnehmen bzw. empfinden, und es besteht eine Reaktionsflexibilität ohne Gedankenmuster (ebd., S. 111).

Im menschlichen Kontext zeigen sich jedoch folgende Schwierigkeiten: Menschen reagieren eben nicht ohne Gedankenmuster, ganz im Gegenteil. Entscheidungsprozesse wie die Generierung von Lösungsalternativen und deren Bewertung und Beurteilung unterliegen mehreren Biases: der selbstdienlichen Wahrnehmung, der Anker-Anpassungsheuristik, der Suche nach Mustern und dem sozialen Einfluss. Diese Biases können nach Bonabeau gemildert werden durch (1) Reichweite: Das Ganze wird größer und damit ebenso die Wahrscheinlichkeit von neuen Positionen; (2) Aggregation: Das Ganze ist gleich der Summe der Teile, Ergebnisse sind der statistische Durchschnitt von Schätzungen; (3) Selbstorganisation: Durch Interaktion wird das Ganze mehr als seine Teile, es entsteht ein (großer) Mehrwert durch Interaktion (kleiner) einzelner Beiträge (Bonabeau 2009, S. $46 \mathrm{ff}$.).

Für menschliche „Schwärme“ bleiben aber weitere Punkte schwierig: Wie gehen Menschen mit dem Kontrollverlust über das Kollektiv bzw. mit der Unvorhersehbarkeit seiner Entscheidungen um? Wie wird die Verantwortlichkeit bei kollektiven Entscheidungen gestaltet? Wie kann eine gute Balance aus Diversität und Expertentum gewährleistet werden? Wie gestaltet sich die Frage nach geistigem Eigentum? Und vor allem: Nach welchen Motivationen gesellt sich ein Individuum zu einem Schwarm? Monetäre Anreize, persönliche Anerkennung, das Bedürfnis, Wissen zu teilen, oder das Gefühl einer gewissen Bürger- oder Mitgestaltungspflicht? 
Der Schritt vom Chaos in Gruppen zu Weisheit von Gruppen liegt nach Bonabeau demnach in der Zusammensetzung der Gruppe aus diversen, unabhängigen und halbwegs informierten Personen sowie in einer gewissen Form von Design der Interaktion. Dieses hat sicherzustellen, dass die Stimmkraft der einzelnen Teilnehmer ausgeglichen wird, Vereinbarungen für Entscheidungen getroffen werden (viele Beiträge mit einer Entscheidung oder viele unabhängige Entscheidungen) und der Prozess handhabbar für alle Beteiligten bleibt (Bonabeau 2009, S. 51).

Der Mensch auf seinem aktuellen Entwicklungsstand scheint also eher dazu geeignet, in überschaubaren Teams und Gruppen intelligent zu arbeiten als in großen Kollektiven. Hierzu entsteht in den letzten Jahren eine bemerkenswerte Forschungsreihe, die im nächsten Kapitel diskutiert wird.

\subsection{Kollektive Intelligenz}

Der französische Philosoph Pierre Lévy entwarf 1994 in seinem Klassiker „L'intelligence collective: pour une anthropologie du cyberspace“ das Zukunftsbild von Wissensgesellschaften, in denen wir Menschen uns neu zu erfinden und aufzustellen haben. Nach Lévy geht es neben technologischen Erfindungen um die Erneuerung der sozialen Bindungen über die Beziehung zum Wissen, d.h. in Prozessen des Lernens voneinander, der Entwicklung einer kollektiven Einbildungskraft und Intelligenz und der synergetischen Nutzung von Kompetenzen. Kollektive Intelligenz sei kein rein kognitives Objekt, sondern hat die Bedeutung vom Wissen um den anderen. Es geht um einen Prozess des Wachsens, der Ausdifferenzierung und der gegenseitigen Befruchtung von Einzelwesen unter ständiger Reflektion: „Lernen wir uns erkennen, um miteinander zu denken“ (Lévy 1997, S. 33). Diese verteilte Intelligenz beruht auf gegenseitiger Anerkennung und Bereicherung, der Aufwertung menschlicher Unterschiede und entsprechenden Kommunikationstechnologien. Es geht nicht um Organisationen von dummen, programmierten Individuen ohne Vision des Ganzen, die blind auf automatische Handlungen zurückgreifen, sondern um ständige Reflexion, auch der Ordnung einer Organisation (ebd., S. 26ff.).

16 Jahre später veröffentlichten Forscher/innen vom MIT Center for Collective Intelligence und der Carnegie Mellon University, Pittsburgh, eine bemerkenswerte Studie. Zum ersten Mal konnten die philosophischen Gedanken Pierre Lévys wissenschaftlich nachvollzogen werden. Anita Woolley und ihre Kolleg/innen wollten 2010 wissen, ob das, was seit mehr 100 Jahren für Individuen erforscht wird, auch für Gruppen existiert: eine Allgemeine Intelligenz, die über die unterschiedlichsten Aufgaben hinweg sichtbar ist und Leistungsvermögen für anstehende Aufgaben vorhersagen kann (Woolley et al. 2010). Hierfür ließen die Forscher/innen insgesamt 700 Teilnehmer/innen in fast 200 Teams à 3-5 Mitgliedern verschiedenste Gruppenaufgaben bearbeiten.

Über den positiven Zusammenhang der jeweiligen Leistungen eines Teams bei den verschiedenen Aufgaben konnten die Forscher/innen zeigen, dass er existiert: ein Faktor Allgemeine Kollektive Intelligenz. Dieser erklärte ca. 43-44\% aller Unterschiede in den Leistungen der verschiedenen Teams, ähnlich wie bei Individuen, deren Allgemeine Intelligenz für 30-50\% der individuellen kognitiven Leistungsunterschiede verantwortlich ist. Anders als die durchschnittliche oder maximale 


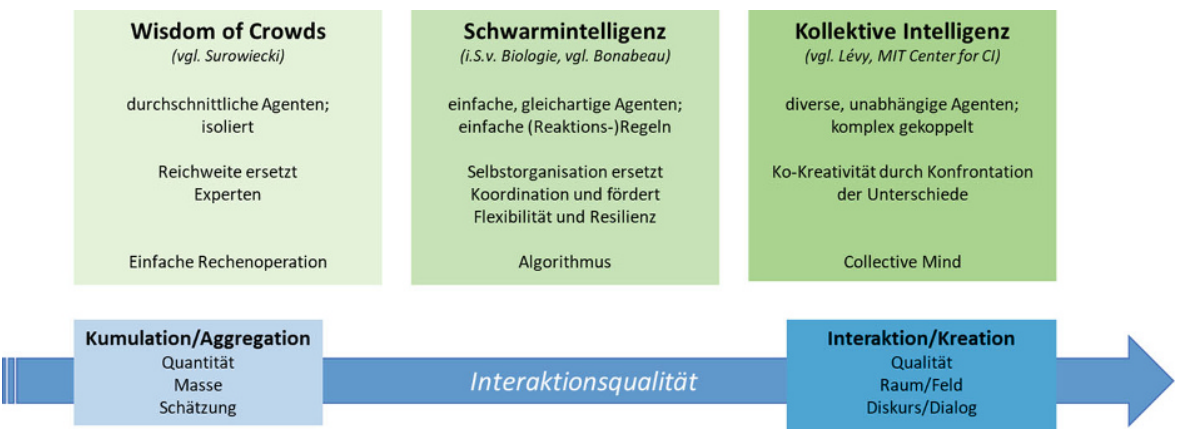

Abb. 1 Konzepte Kollektiver Intelligenz. (Eigene Darstellung)

Intelligenz der Einzelpersonen in der Gruppe konnte der Faktor der Kollektiven Intelligenz die Gruppenleistung vorhersagen. Kollektive Intelligenz wird daher als eine Eigenschaft der Gruppe selbst gesehen: Sie besitzt Vorhersagekraft jenseits dessen, was über die Fähigkeiten der Individuen erklärt werden kann.

Auf der Suche nach den Einflussfaktoren dieser Kollektiven Intelligenz erwiesen sich die naheliegenden Vermutungen als Irrtum. Gruppenzusammenhalt, Motivation oder Zufriedenheit waren nicht verantwortlich für den Unterschied an Kollektiver Intelligenz in den Gruppen. Auch individuelle Intelligenz hatte hier nur einen moderaten Einfluss und konnte die Unterschiede in den Gruppenleistungen nicht vorhersagen. Letzten Endes stieß man auf drei wesentliche Einflussfaktoren für die Kollektive Intelligenz: die Ausgeglichenheit der Wortmeldungen in der Gruppe (,equal distribution of conversational turn-taking “), die Fähigkeit der Teilnehmer/ innen, die Gefühlslage ihres Gegenübers zu erkennen (,, social sensitivity“ oder soziale Wahrnehmung) und der Anteil Frauen (Woolley et al. 2010, S. 688). Letzteres führte man auf die - durch Messungen bestätigte - höhere soziale Sensitivität bei den teilnehmenden Frauen zurück.

Die MIT-Studie hat eindrucksvoll belegt, dass die individuelle Intelligenz, die maßgeblichen Einfluss auf Einzelleistungen hat, kaum Einfluss auf die Leistung einer Gruppe hat, die vor derselben Aufgabe steht. Eine Gruppe von Intelligenzbestien ist also noch lange nicht smart. Die Individuen müssen auch eine Kultur für das Miteinander finden, die die Egos in Schach hält und die Introvertierten zum Beitrag ermutigt. So werden die individuellen Stärken zu einem Mehr integriert statt zu einem Weniger abgeschliffen. Eine Gruppe mit geringeren Einzel-IQs, aber hoher Qualität in der Begegnungskultur kann weitaus smarter werden als eine Gruppe von Genies.

Während also die Weisheit der Vielen ohne jegliche soziale Interaktion operieren kann (und muss) und Schwarmintelligenz bereits (aber auch nur) mit sehr einfachen kognitiven Mustern entstehen kann, hat menschliche Kollektive Intelligenz also immer mit sozialer Interaktion von diversen, unabhängigen Individuen zu tun sowie mit bewusster Gestaltung eben dieser Interaktion (s. Abb. 1). 


\section{Intelligenz im Gefühl: Soziale Wahrnehmung und Psychologische Sicherheit}

Auf der Suche nach dem perfekten Team ist auch Google auf die MIT-Studie aufmerksam geworden (Projekt Aristotele 2012-2015). Innerhalb von vier Jahren und mit eigenen Daten von 180 Teams kamen sie ebenfalls zu dem Schluss: Für die Teamperformance zählt nicht das Wer, sondern das Wie. Doch statt dies wie die Forscher/innen des MIT durch das Konzept der Sozialen Wahrnehmung zu erklären, bedienten sie sich des Konzepts der Psychologischen Sicherheit (Duhigg 2016).

Es liegt auch nahe, zu vermuten, dass soziale Einflussfaktoren, die als förderlich für Gruppenlernen und Gruppenleistung bestätigt wurden, wie z. B. Gruppenzusammenhalt, Gruppenzufriedenheit oder Psychologische Sicherheit, generell mit Kollektiver Intelligenz in Zusammenhang stehen bzw. diese fördern, wenn bereits soziale Wahrnehmung als einer der wesentlichen Einflussfaktoren bestätigt wurde. Erstaunlicherweise zeigen entsprechende Studien aber wiederholt, dass dies nicht der Fall ist. Kollektive Intelligenz und Psychologische Sicherheit, gemessen an den von Edmondson (1999) etablierten subjektiv abgefragten Items, zeigen sich als unabhängig voneinander und korrelieren nicht. Die Förderung von Psychologischer Sicherheit steigert nicht die Kollektive Intelligenz eines Teams. Die gesteigerte Leistungsfähigkeit der Google-Teams hatte demzufolge nicht unbedingt mit Kollektiver Intelligenz zu tun. Die Frage nach dem größeren Zusammenhang der unterschiedlichsten Konzepte und Forschungen liegt nahe. Im Folgenden wird dieser anhand der neuesten Studienlage dargestellt.

\subsection{Psychologische Sicherheit und Kollektive Intelligenz}

Ursprüngliche Konzepte der Psychologischen Sicherheit (Kahn 1990) formulierten diese als individuelle Wahrnehmung oder individuelles Gefühl von Sicherheit durch vertrauensvolle, unterstützende zwischenmenschliche Beziehungen, das dazu beiträgt, sich selbst ohne Angst vor negativen Konsequenzen zu zeigen. Amy Edmondson (1999) hob dieses Gefühl auf die Teamebene und beschrieb Psychologische Sicherheit als ein Teamklima, im Detail als ein ,,von den Mitgliedern des Teams geteilter Glaube, dass es im Team sicher genug ist, um zwischenmenschliche Risiken eingehen zu können“ (Edmondson 1999, S. 350). Erhoben wird Psychologische Sicherheit in wissenschaftlichen Untersuchungen seitdem meist über Abfrage von sieben von Edmondson definierten Kriterien: (1) Respekt, (2) gegenseitiges Interesse, (3) Zulassen individueller Meinungen, (4) positive Intentionen, (5) gesunde Fehlerkultur, (6) Eingehen von Risiken sowie (7) grundsätzlich wohlmeinende Haltungen (Edmondson 1999, S. 360, 363).

Vielfältige positive Auswirkungen von Psychologischer Sicherheit wurden seitdem in zahlreichen Studien bestätigt, u.a. mehr persönliche Kommunikation und Wissensteilung, offenes Feedback, offene Ansprache von Problemen und Differenzen sowie die Reflexion und Lösung derselben oder kreatives Denken unter Eingehen von Risiken (Newman et al. 2017; Edmondson und Lei 2014). Nicht bestätigt wurde hingegen, dass Psychologische Sicherheit vermehrt zu kritischem Denken führt 


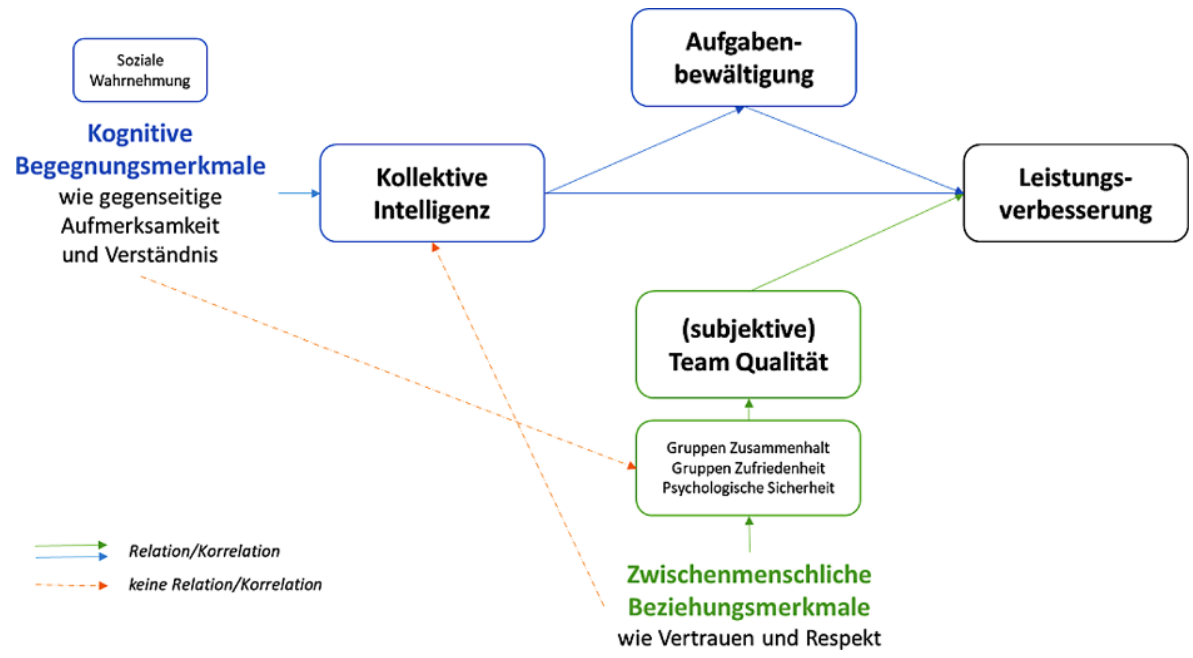

Abb. 2 Relationen zwischen Kollektiver Intelligenz, Team-Qualität, Aufgabenbewältigung und Leistungsverbesserung. (Eigene Darstellung in Anlehnung an Woolley und Aggarwal 2019, S. 35)

(Newman et al. 2017, S. 527), ein wichtiger Punkt, den ich später noch aufgreifen werde.

Ist Psychologische Sicherheit also eventuell eine notwendige, aber keine hinreichende Bedingung für Kollektive Intelligenz? Woolley und Aggarwal (2019) konnten einen anderen Zusammenhang finden, indem sie den Einfluss von Kollektiver Intelligenz auf drei, aus unterschiedlichen Disziplinen stammende Dimensionen des Gruppenlernens untersuchten: Verbesserung der Ergebnisse, Synergien in der Aufgabenbearbeitung und positives zwischenmenschliches Klima durch Gruppenprozesse (Woolley und Aggarwal 2019, S. $10 \mathrm{ff}$.):

- Leistungsverbesserung drückt sich in durch Erfahrung generierten Lernkurven aus und kann an verbesserten Ergebnissen gemessen werden.

- Aufgabenbewältigung drückt sich in der Art der kollektiven Informationsbearbeitung wie z. B. Informationseinholung und -teilung oder Entscheidungsprozessen aus und wird in Form von kognitiver Synergie gemessen: Eine starke kognitive Synergie liegt vor, wenn das Gruppenergebnis größer ist als das Ergebnis des besten einzelnen Mitglieds - klassisch $1+1=3$.

- Subjektive Qualität der Teaminteraktion drückt sich in aktivem Feedback und Hinterfragen aus, sodass Korrekturen vorgenommen werden können, wenn nötig. Messungen können immer nur über abgefragte subjektive Bewertungen vorgenommen werden.

Woolley und Aggarwal konnten zeigen, dass Kollektive Intelligenz einen direkten Einfluss auf die ersten beiden Dimensionen hat, aber nicht auf die subjektiv wahrgenommene Qualität der Teaminteraktion. Letztere hatte aber ihrerseits einen Einfluss auf die Leistungsverbesserung. Sie schließen daraus, dass Lernen in Gruppen und das Funktionieren von Gruppen generell über zwei Pfade unterstützt wird: einen sozio-emotionalen sowie einen kognitiven (s. Abb. 2). Den Autorinnen zufolge 
wurde diese Trennung bereits in Studien zu Team-Effektivität oder Team-Kognition bestätigt und ist aktuell auch in Studien zu Empathie zu finden. Ebenso kann eine ähnliche Dualität in den Konzepten zu kollektivem Wissen aufgezeigt werden (Feldhusen 2014, S. 111 ff., 199 ff.). Woolley und Aggarwal (2019) zeigen darüber hinaus, wie die beiden Ebenen der Aufgabenbewältigung und der subjektiven Team-Qualität in Zusammenhang stehen. Sie korrelieren zwar nicht, stellen aber gleichwertige, sich kompensierende Beiträge zur Leistungsverbesserung in Teams dar. Dies konnte bislang für Gruppen bestätigt werden, die über eher kurze Zeiträume zusammenarbeiten. Ob sich Synergie-Effekte bei lang zusammenarbeitenden Teams ergeben, bleibt Gegenstand weiterer Forschung.

\subsection{Sozio-emotionale versus kognitive Pfade zu Gruppenleistungen}

Der sozio-emotionale Pfad ist recht klar zu greifen. Er bezieht sich auf zwischenmenschliche Faktoren wie Zusammenhalt, Psychologische Sicherheit, Gruppenatmosphäre oder Zufriedenheit, d.h. darauf, was Gruppenmitglieder in Bezug aufeinander fühlen. Sozio-emotionale Faktoren generieren Lernen durch eine Erweiterung des Verhaltensrepertoires und Verbesserung des Gruppenprozesses (ebd., S. 15). Der sozio-emotionale Pfad, d.h. auch Psychologische Sicherheit, kann daher gesehen werden als die Voraussetzung dafür, dass sich jeder Einzelne einbringt, mit seinen Fähigkeiten, seinem Wissen, seinen Beobachtungen, Meinungen und Ideen.

Der kognitive Pfad ist komplexer, da es hier um die emergente Wirkung von Interaktionen geht, die als systemischer Prozess beides enthält: Top-down-Prozesse, d.h. Wirkungen vom Team auf das Individuum, als auch Bottom-up-Prozesse, d.h. Wirkungen vom Individuum auf das Team. Es geht darum, wie die eingebrachten Ressourcen miteinander kombiniert und bearbeitet werden, um zu einem Ergebnis zu kommen. Neben der absoluten Menge an interpersonaler Kommunikation geht es nach Woolley um Ausgeglichenheit in der Partizipation und um eine moderate kognitive Diversität: Bei zu hoher Diversität besteht die Gefahr, dass keine fruchtbare Kommunikation möglich ist, bei zu geringer Diversität könnte ein Mangel an Perspektivenvielfalt herrschen (Woolley et al. 2015, S. 422). Insbesondere geht es aber um eine Reihe an zwischenmenschlichen Fähigkeiten (Woolley und Aggarwal 2019; Woolley et al. 2015):

- soziale Wahrnehmung, d.h. die Fähigkeit, Emotionen in anderen wahrzunehmen und zu beurteilen, wobei hier der niedrigste Einzelwert den Gruppenwert bestimmt (gemessen im Reading The Mind inThe Eyes Test),

- gegenseitige Aufmerksamkeit und Verständnis (gemessen an Gleichzeitigkeit von Gesichtsausdrücken),

- Antizipation der Entscheidungen anderer, um die eigenen darauf abstimmen zu können,

- nicht aber: Zustimmungsbereitschaft oder Pflichtbewusstsein.

Über diese Fähigkeiten kommt eine Gruppe auf eine weitaus höhere Interaktionsebene, auf der über kognitive Synergiegewinne die Leistung größer ist als die Summe der möglichen Einzelleistungen. Die Gruppe kann aus den bestehenden Erfahrungen der Teilnehmer weitaus mehr und qualitativ höhere Informationen für die 
anliegende Fragestellung generieren, u. a. weil auch subtile, nonverbale Hinweise mit einfließen können und die Teilnehmenden sich individuell immer wieder auf die Gesamtsituation abstimmen. Es zeigte sich, dass Kollektive Intelligenz bereits früh am Beginn einer Gruppe entsteht, auch unter fremden Personen, und relativ stabil erscheint. Der positive Leistungsunterschied im Vergleich zu anderen Gruppen zeigt sich erst im Laufe mehrerer Arbeitsrunden, sodass sich der Mehrwert hauptsächlich über schnelleres Lernen generiert. Diese Wirkungen wurden nicht nur in der face-toface-Interaktion, sondern auch in der Online-Zusammenarbeit nachgewiesen (Woolley et al. 2015, S. 421). Der kognitive Pfad, d.h. auch Soziale Wahrnehmung, kann somit gesehen werden als ein Indikator für die Fähigkeit, sich auf das Gegenüber einzulassen, das Ganze im Blick zu haben. Es ist eine Qualität von Wahrnehmung des Anderen und von Begegnung mit dem Anderen, um dann in einem kritischen Denkprozess zu einer neuen, besseren Lösung zu kommen.

Woolley und Aggarwal (2019) erinnern angesichts ihrer Ergebnisse an die klassische Differenzierung von Aufgaben- und Beziehungsorientierung, doch die vorliegende Unterscheidung geht darüber hinaus.

Sozio-emotionale Faktoren beschreiben, wie sicher und wohl sich der Einzelne fühlt, ob er als Person gesehen und akzeptiert wird. In Studien zu Team-Qualität, Psychologischer Sicherheit und anderen sozio-emotionalen Konzepten beruhen die gemessenen Parameter auf individuellen Abfragen und sagen demzufolge etwas darüber aus, wie sich im Durchschnitt der Einzelne im Team aufgehoben fühlt oder was er darüber denkt, wie er von anderen wahrgenommen wird. Dies ist jedoch nicht nur eine Folge der Gruppensituation, sondern auch entscheidend geprägt durch die psychologische Vor-Verfassung des Einzelnen. Es kann davon ausgegangen werden, dass angelegte Persönlichkeitsstrukturen und Selbstwahrnehmungen Einfluss auf die Messwerte von Psychologischer Sicherheit haben und diese sich nicht entsprechend der Definition allein auf die Wahrnehmung des Gruppenklimas beziehen. In demselben Klima fühlt sich die eine sicher, der andere weniger. Die Wahrnehmungen, auf die sich diese Konzepte stützen, sind demnach eher ich-zentriert.

Soziale Wahrnehmung kann dagegen unabhängig davon stattfinden, ob das Klima vom Einzelnen als positiv empfunden wird oder ob er sich psychologisch sicher wähnt. Es geht nicht nur darum, wie sich der Einzelne fühlt, sondern inwieweit er selbst anderen Raum gibt und sich selbst im Ganzen abstimmt. Es geht um die interessierte Wahrnehmung anderer Personen und die Einbindung von deren Perspektiven und Äußerungen in den eigenen Meinungsprozess unter Beachtung einer Gesamtsituation. Es geht hier zunächst nicht darum, sich und seine Meinung einzubringen, sondern darum, seine Perspektive zu erweitern durch die Wahrnehmung anderer. Für das mit diesen Faktoren verwandte Konzept des Perspective Taking, das das intuitive Erkennen von Gedanken, Gefühlen und mentalen Zuständen anderer Personen beschreibt (Epley \& Caruso 2009), konnten folgende Vorrausetzungen in Studien belegt werden: Es braucht die Bereitschaft durch bewusste Aktivierung, die Überwindung des eigenen Egos sowie die aktive Einholung von Informationen über mein Gegenüber (Lozano et al. 2006). Die Wahrnehmungen, auf die sich diese Konzepte stützen, sind demnach eher situations-zentriert oder selbst-transzendierend. Letztere Bezeichnung lehnt sich an das Konzept des ,self-transcending knowledge“ 
(Scharmer 2001) an, das sich auf die Überschreitung der eigenen Perspektive, des eigenen Denk- und Wissensraums bezieht.

Diese Fähigkeit zur selbst-transzendierenden Wahrnehmung hängt nicht unbedingt zusammen mit dem Gefühl von Sicherheit oder Aufgehobenheit. Im Gegenteil, sich wohl und sicher zu fühlen, kann die Falle bergen, andere Perspektiven eben nicht wahrzunehmen, sie nicht wahrnehmen zu wollen oder sie als nicht relevant zu bewerten. Der Schritt aus der eigenen Comfort-Zone wird nicht notwendig, und der Schritt zum Group Think (Janis 1972) liegt nahe. Hier schwächt der Wunsch nach Harmonie das Urteilsvermögen und führt zu Verlust an Kreativität, Einzigartigkeit und unabhängigem Denken. In diese Richtung mag es zu interpretieren sein, dass eine größere Tendenz zu unethischem Verhalten als eine - wenn auch einzige negative Wirkung von Psychologischer Sicherheit gezeigt werden konnte (Newman et al. 2017, S. 528). Hingegen waren Anzeichen von Harmoniebedürfnis wie Zustimmungsbereitschaft oder Pflichtbewusstsein nicht korrelierend mit Kollektiver Intelligenz (Woolley und Aggarwal 2019, S. 26).

In einer Gruppe, in der jeder einzelne aktiv die Aufmerksamkeit auf den und die anderen legt, in der Aktionen von Beitrag und Unterordnung sich abwechseln, kann ein Collective Mind (Weick und Roberts 1993) entstehen. Hier generieren die drei Aktionen des Beitrags (der Einzelnen zum Ganzen), der Repräsentation (des Gesamtsystems im Blick des Einzelnen) und der Unterordnung (Verbindung der Aktionen des Einzelnen mit dem Gesamtsystem) ein miteinander geteiltes Feld mit transindividueller Qualität (ebd., S. 363), also einen intelligenten kollektiven Denk-, Kommunikations- und Handlungsraum, der über den Einzelnen hinausweist und aus dem heraus Gruppen intelligenter handeln können als die Summe der einzelnen Mitglieder. Bereits von Bohm wurde auf die für solche Prozesse des TeamDialogs entscheidenden Voraussetzungen hingewiesen: Aufmerksamkeit im Prozess des Zuhörens, Mitgefühl und eine Haltung der Nicht-Wertung. Für Bohm bedeutet Intelligenz, dass man seine Annahmen nicht verteidigt, sondern offen bleibt im Prozess des gemeinsamen Denkens (Bohm 1996, S. 34).

\subsection{Beziehungsqualität und Begegnungsqualität}

Die beiden beschriebenen Pfade und Konzepte liegen so nahe beieinander, dass die analytische Trennung oft schwer fällt und die Konzepte drohen, miteinander vermischt zu werden. Psychologische Sicherheit wird in wissenschaftlichen Studien üblicherweise mit den von Edmondson (1999) erhobenen sieben Kriterien abgefragt, die über subjektive Gefühle Auskunft geben. In weiteren Diskussionen wird Psychologische Sicherheit bereits ausgedehnt auf Konzepte des aktiven Zuhörens, der Unvoreingenommenheit oder Inklusion aller Teilnehmer. Diese gehören jedoch nicht zu dem Testkatalog und sind eher dem kognitiven Pfad der Sozialen Wahrnehmung zuzuordnen. In Abfrageerhebungen - wie auch in der Realität der Gruppenarbeit besteht ein wesentlicher Unterschied zwischen den Aussagen: „Ich glaube, ich werde im Team nicht für meine Meinungen bewertet, ich fühle mich sicher“ und „Ich bewerte die Meinungen anderer nicht".

In der weiteren Erforschung ist daher eine saubere Trennung der Konzepte geboten, d.h. zwischen ich-zentrierten Erhebungen, die Auskunft darüber geben, wie sich 
der Einzelne in Beziehung setzt zu anderen, also das Ich in Beziehung zu anderen sieht (,Ich fühle mich sicher“), und den situations-zentrierten oder selbst-transzendierenden Erhebungen, die Auskunft darüber geben, wie weit er von eben diesem Ich Abstand nehmen kann und das Gegenüber wahrnimmt, also in Begegnung geht (,Ich nehme beim anderen Sicherheit wahr“). Im ersten Fall kann man von gefühlter Beziehungsqualität sprechen, im zweiten von praktizierter Begegnungsqualität.

\section{Auf dem Weg zu smarten Organisationen}

Agile Zusammenarbeit von Teams und Gruppen wird in komplexen und unsicheren Umfeldern immer wichtiger und zum Erfolgsfaktor. Gerade bei sich immer wieder ändernden Anforderungen und Aufgabenstellungen ist es essentiell, als Team schnell zusammenarbeiten zu können und durch optimale Aufnahme und Verarbeitung von Informationen im Hinblick auf die aktuelle Aufgabe hierin laufend besser zu werden.

In der Zusammenarbeit entfaltet das Wie des Miteinanders signifikant mehr Wirkung als das Wer der Zusammensetzung. Dieses Wie von Gruppen-Interaktionen hat offensichtlich zwei voneinander unabhängige Dimensionen: eine sozio-emotionale oder individual-psychologische und eine sozial-kognitive. Beide spielen eine zentrale und gleichberechtigte Rolle - sowohl das individuelle Empfinden, ausgedrückt z. B. über Psychologische Sicherheit, als auch die Fähigkeit des Geistes, sich auf andere einzulassen, ausgedrückt z. B. über Soziale Wahrnehmung. Ersteres scheint wichtig, um grundsätzlich gut zusammenarbeiten zu können, Letzteres ist wichtig, um darin besser zu werden und auch auf neue Situationen reagieren zu können.

Psychologische Sicherheit bewegt Gruppenmitglieder zur Partizipation, zum „Beitrag des Eigenen“, doch zu viel Behagen und Sicherheitsgefühl im Team kann auch lähmend für geistige Beweglichkeit sein. Soziale Wahrnehmung befähigt demgegenüber zu „Teilnahme“, dem Annehmen des Anderen, einem zentralen Faktor für Kollektive Intelligenz, die sich wiederum durch bessere Koordination, Aufgabenbewältigung und vor allem durch erhöhte Lerngeschwindigkeit im Team ausdrückt. Damit bekommen Kollektive Intelligenz sowie ihre Einflussfaktoren Soziale Wahrnehmung und ähnliche Konzepte wie Perspective Taking gerade in dynamischen und volatilen Umfeldern eine enorme Bedeutung für die Gestaltung von Teams und Organisationen.

Kollektive Intelligenz entsteht offensichtlich nicht allein durch Quantität, d.h. die schiere Masse, die einen mathematischen Durchschnitt näher an die Wahrheit bringt. Und intelligente Lösungen entstehen offenbar auch nicht allein durch ein Mehr an Daten oder optimierten Prozessen. Vielmehr entsteht Kollektive Intelligenz durch die Qualität menschlicher Begegnung und ihres Design- bzw. Organisationsprozesses. Entscheidend ist, wie wir uns und anderen zuhören, Unterschiede wahrnehmen und verarbeiten, aufeinander eingehen, uns auf neue Perspektiven einlassen, miteinander Lösungen verhandeln, uns und dem Prozess der Interaktion Aufmerksamkeit schenken. Wie eingangs zitiert, sieht Gary Hamel die Führungskräfte der Zukunft als Architekten sozialer Systeme. Dies bedeutet für die meisten Mitarbeiter/innen und Führungskräfte einen tiefgreifenden Reife- und Entwicklungsprozess hin zu einer 
Haltung, die dem Gegenüber mindestens die gleiche Bedeutung beimisst wie dem Selbst.

Funding Open access funding provided by Danube University Krems University for Continuing Education.

Open Access Dieser Artikel wird unter der Creative Commons Namensnennung 4.0 International Lizenz veröffentlicht, welche die Nutzung, Vervielfältigung, Bearbeitung, Verbreitung und Wiedergabe in jeglichem Medium und Format erlaubt, sofern Sie den/die ursprünglichen Autor(en) und die Quelle ordnungsgemäß nennen, einen Link zur Creative Commons Lizenz beifügen und angeben, ob Änderungen vorgenommen wurden.

Die in diesem Artikel enthaltenen Bilder und sonstiges Drittmaterial unterliegen ebenfalls der genannten Creative Commons Lizenz, sofern sich aus der Abbildungslegende nichts anderes ergibt. Sofern das betreffende Material nicht unter der genannten Creative Commons Lizenz steht und die betreffende Handlung nicht nach gesetzlichen Vorschriften erlaubt ist, ist für die oben aufgeführten Weiterverwendungen des Materials die Einwilligung des jeweiligen Rechteinhabers einzuholen.

Weitere Details zur Lizenz entnehmen Sie bitte der Lizenzinformation auf http://creativecommons.org/ licenses/by/4.0/deed.de.

\section{Literatur}

Bohm, D. (1996). On Dialogue L. Nichol. London, New York: Routledge.

Bonabeau, E. (2009). Decisions 2.0: the power of collective intelligence. MIT Sloan Management Review, 50 (2) (Winter 2009), 45-52.

Bonabeau, E., \& Meyer, C. (2001). Swarm intelligence. A whole new way to think about business. Harvard Business Review, May, 107-114.

Cândido, C., \& Santos, S. (2015). Strategy implementation: what is the failure rate? Journal of Management \& Organization, 21(2), 237-262.

Duhigg, C. (2016). What Google learned from its quest to build the perfect team. New York Times, 2016,02.02. https://www.nytimes.com/2016/02/28/magazine/what-google-learned-from-its-questto-build-the-perfect-team.html. Zugegriffen: 1. Apr. 2021.

Edmondson, A. (1999). Psychological safety and learning behavior in work teams. Administrative Science Quarterly, 44, 350-383.

Edmondson, A., \& Lei, Z. (2014). Psychological safety: the history, renaissance, and future of an interpersonal construct. The Annual Review of Organizational Psychology and Organizational Behavior, 1 , 23-43.

Epley, N., \& Caruso, E. M. (2009). Perspective taking: misstepping into others' shoes. In K. D. Markman, W.M.P. Klein \& J. A. Suhr (Hrsg.), Handbook of imagination and mental simulation (S. 295-309). New York: Psychology Press.

Feldhusen, B. (2014). Organizing future: an integrated framework for the emergence of collective selftranscending knowledge. Dissertation. Wien: Wirtschaftsuniversität Wien.

Hamel, G. (2009). Moon shots for management. Harvard Business Review, February, 91-98.

Hofert, S. (2018). Agiler führen: Einfache Maßnahmen für bessere Teamarbeit, mehr Leistung und höhere Kreativität (2. Aufl.). Springer: Gabler.

Janis, I. (1972). Victims of groupthink: a psychological study of foreign-policy decisions and fiascoes. Oxford: Houghton Mifflin.

Kahn, W. A. (1990). Psychological conditions of personal engagement and disengagement at work. Academy of Management Journal, 33, 692-724.

Lambers, S. (2019). Die meisten Change Projekte scheitern. Managerseminare, 251 (Februar 2019). https:// www.managerseminare.de/ms_Artikel/Veraenderungsmanagement-Die-meisten-Change-Projektescheitern,269249. Zugegriffen: 1. Apr. 2021.

Leimeister, J. M. (2010). Kollektive Intelligenz. Wirtschaftsinformatik, 4, 239-242.

Lévy, P. (1994). L'intelligence collective : pour une anthropologie du cyberespace. Paris: La Découverte.

Levy, P. (1997). Die kollektive Intelligenz: für eine Anthropologie des Cyberspace. Köln: Bollmann.

Lorenz, J., et al. (2011). How social influence can undermine the wisdom of crowd effect. Proceedings of the National Academy of Sciences of the United States of America, 108(22), 9020-9025. 
Lozano, S.C., Hard, M. B., \& Tversky, B. (2006). Perspective taking promotes action understanding and learning. Journal of Experimental Psychology: Human Perception and Performance, 32(6), 1405.

MIT (2021). What is Collective Intelligence? In: Handbook of Collective Intelligence. https://scripts.mit. edu/ cci/HCI/index.php?title=What_is_collective_intelligence\%3F. Zugegriffen: 1. Apr. 2021.

Newman, A., Donohue, R., \& Eva, N. (2017). Psychological safety: a systematic review of the literature. Human Resource Management Review, 27, 521-535.

Scharmer, C.O. (2001). Self-transcending knowledge: sensing and organizing around emerging opportunities. Journal of Knowledge Management, 5(2), 137-150.

Senge, P. (1990). The fifth discipline. New York: Doubleday Business.

Surowiecki, J. (2004). The Wisdom of Crowds: why the many are smarter than the few and how collective wisdom shapes business, economies, societies and nations. New York: Doubleday.

Teece, D. J. (2009). Dynamic capabilities \& strategic management: organizing for innovation and growth. Oxford: Oxford University Press.

Teece, D. J., \& Pisano, G. (1994). The dynamic capabilities of firms: an introduction. Industrial and Corporate Change, 3(3), 537-556.

Teece, D. J., Pisano, G., \& Shuen, A. (1997). Dynamic capabilities and strategic management. Strategic Management Journal, 18(7), 509-533.

Weick, K.E., \& Roberts, K.H. (1993). Collective mind in organizations: heedful interrelating on flight decks. Administrative Science Quarterly, 38, 357-381.

Weinberg, U. (2018). Network Thinking - Was kommt nach dem Brockhaus-Denken? (2. Aufl.). Hamburg: Murmann.

Woolley, A. W., \& Aggarwal, I. (2019). Collective intelligence and group learning. Working paper under review.

Woolley, A.W., et al. (2010). Evidence for a collective intelligence factor in the performance of human groups. Science, 330(6004), 686-688.

Woolley, A. W., Aggarwal, I., \& Malone, T.W. (2015). Collective intelligence and group performance. Current Directions in Psychological Science, 24(6), 420-424.

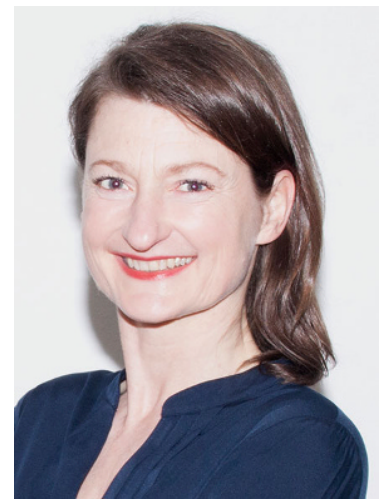

Dr. Birgit Feldhusen Nach 15 Jahren in Konsumgüterindustrie und Beratung ging die Autorin 2010 in die Wissenschaft, um zu erforschen, wie Menschen gemeinsam über sich hinauswachsen können; Promotion zur Emergenz von kollektivem Wissen an der Wirtschaftsuniversität Wien. Sie gründete 2015 das Institut „organizing future“ und etablierte 2018 den Kompetenzbereich „Future Organizations“ an der Donau-Universität Krems, die beide auf Basis von Forschungen u. a. zu Kollektiver Intelligenz vielfältige Formate für neue Perspektiven auf Organisation und Führung bieten. 